\title{
A new method for learning imprecise hidden Markov models
}

\section{Arthur Van Camp and Gert de Cooman}

Ghent University, SYSTeMS

Arthur.VanCamp@UGent.be, Gert.deCooman@UGent.be 


\section{Imprecise hidden Markov models}




\section{Imprecise hidden Markov model}

graphical representation

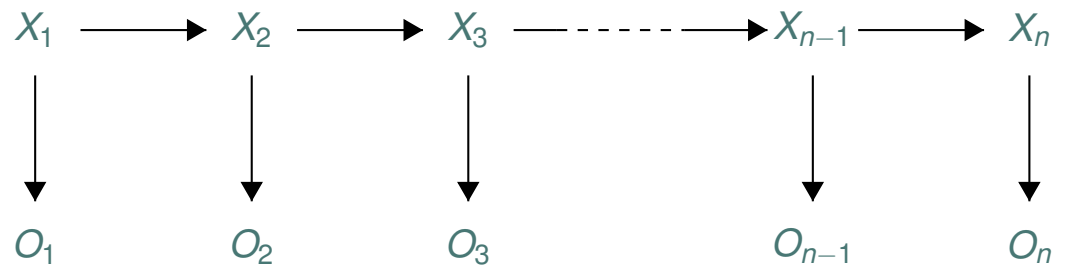




\section{Imprecise hidden Markov model}

\section{random variables}

state variables: HIDDEN

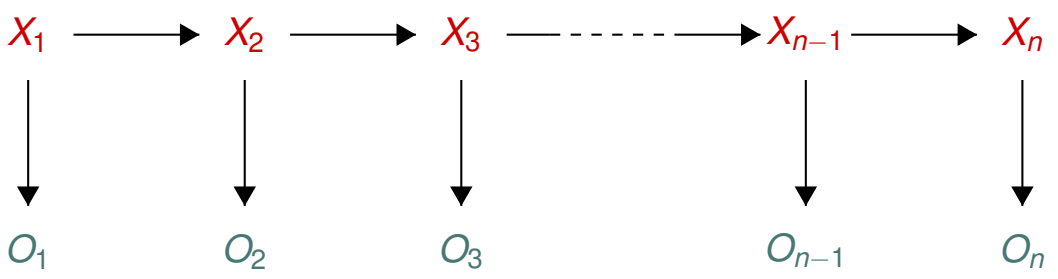




\section{Imprecise hidden Markov model}

\section{random variables}

state variables: HIDDEN

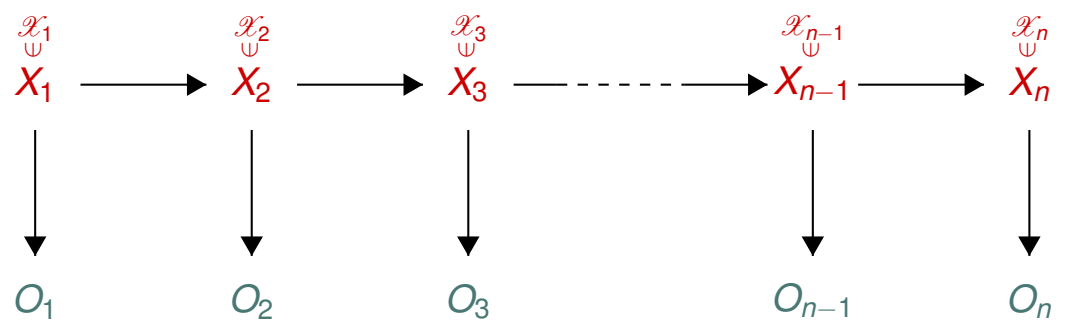




\section{Imprecise hidden Markov model}

\section{random variables}

state variables: HIDDEN

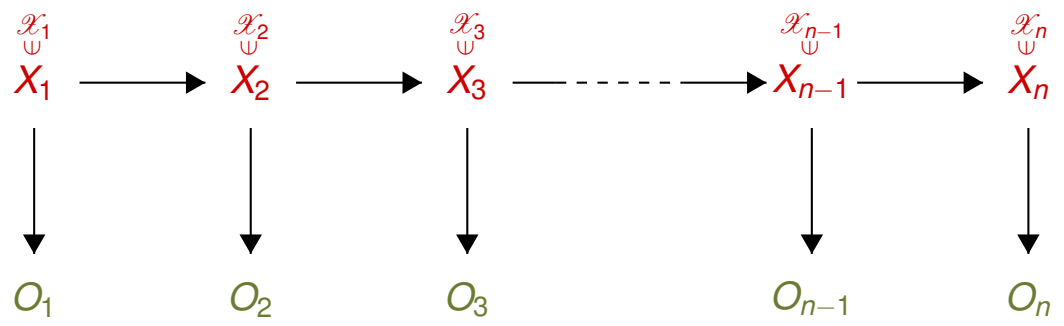

output variables: OBSERVABLE 


\section{Imprecise hidden Markov model}

\section{random variables}

state variables: HIDDEN

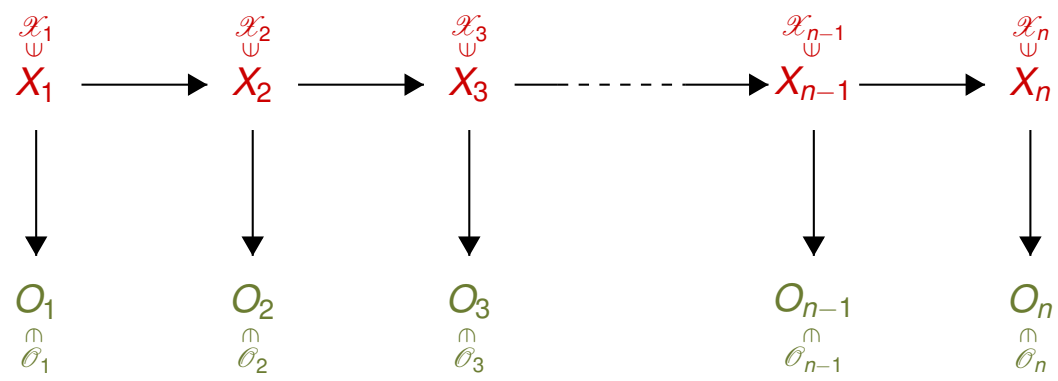

output variables: OBSERVABLE 


\section{Imprecise hidden Markov model}

We consider stationary imprecise hidden Markov models

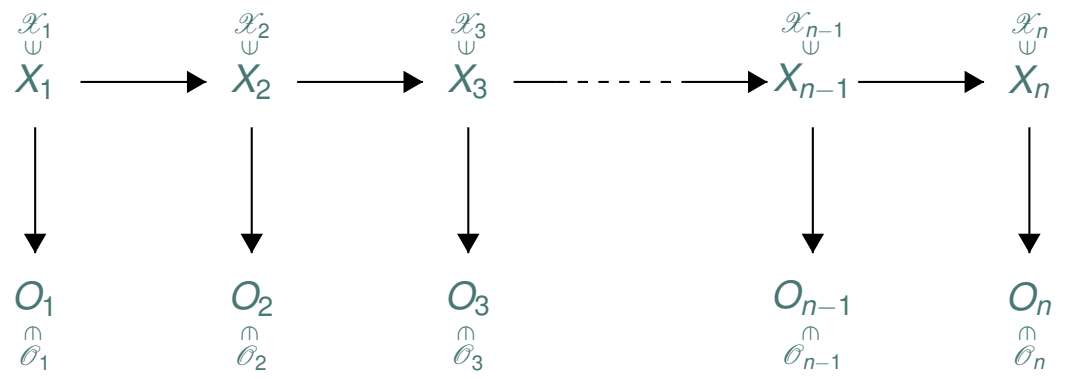




\section{Imprecise hidden Markov model}

We consider stationary imprecise hidden Markov models

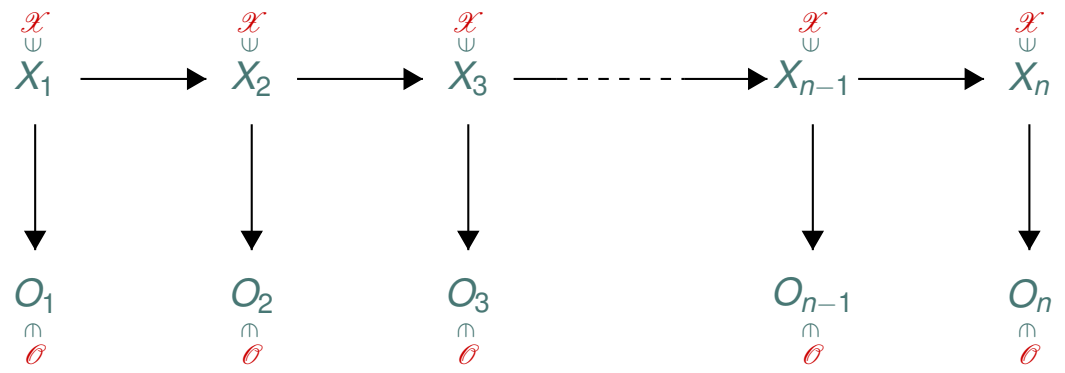




\section{Imprecise hidden Markov model}

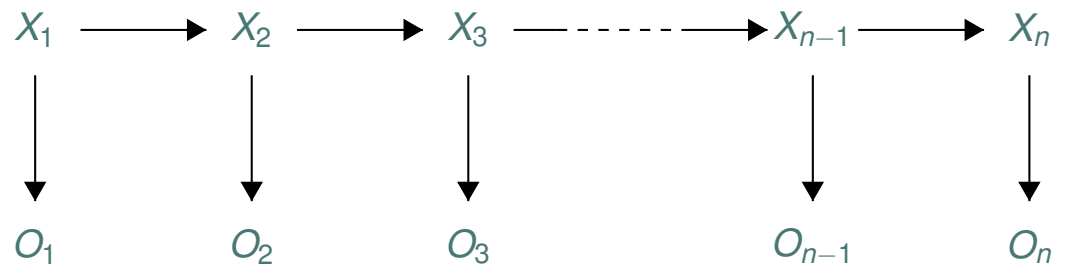




\section{Imprecise hidden Markov model}

local uncertainty models in terms of coherent lower previsions

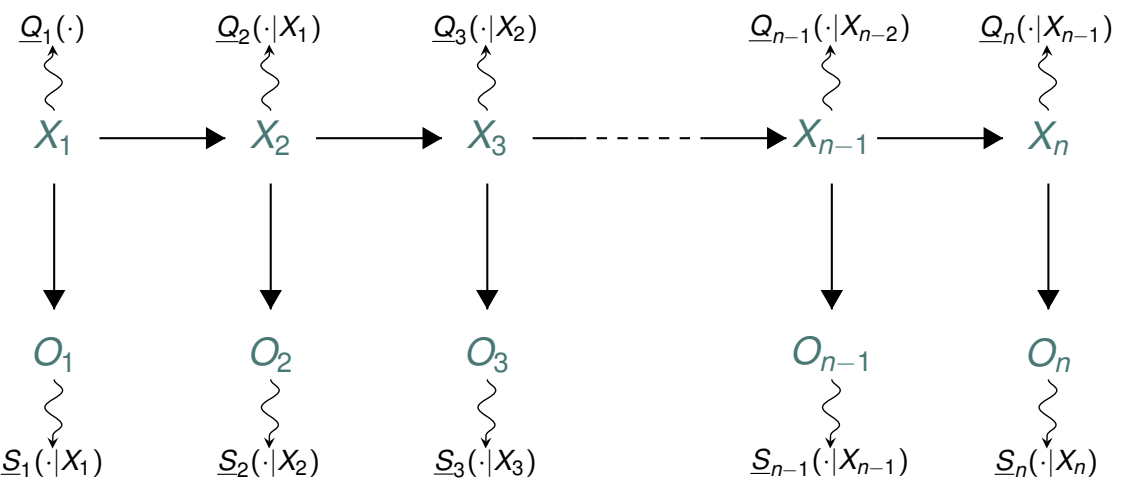




\section{Imprecise hidden Markov model}

local uncertainty models in terms of coherent lower previsions

imprecise transition models

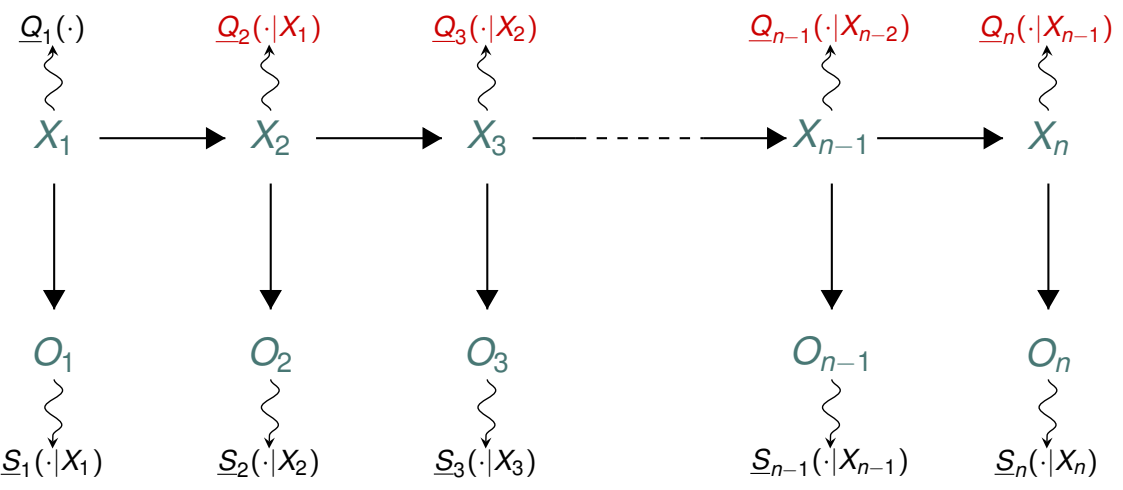




\section{Imprecise hidden Markov model}

local uncertainty models in terms of coherent lower previsions

imprecise transition models

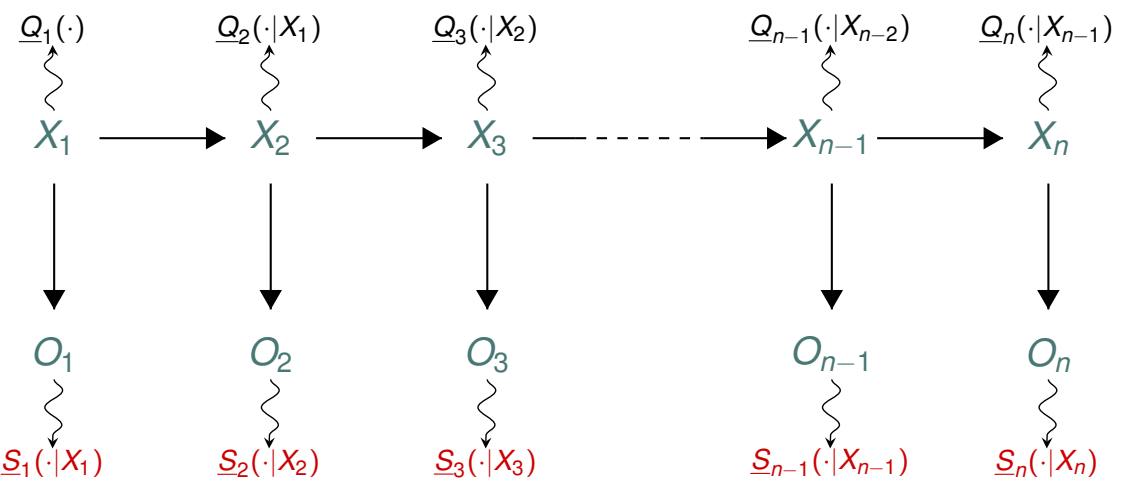

imprecise emission models 


\section{Imprecise hidden Markov model}

local uncertainty models in terms of coherent lower previsions imprecise marginal model

$$
\text { imprecise transition models }
$$
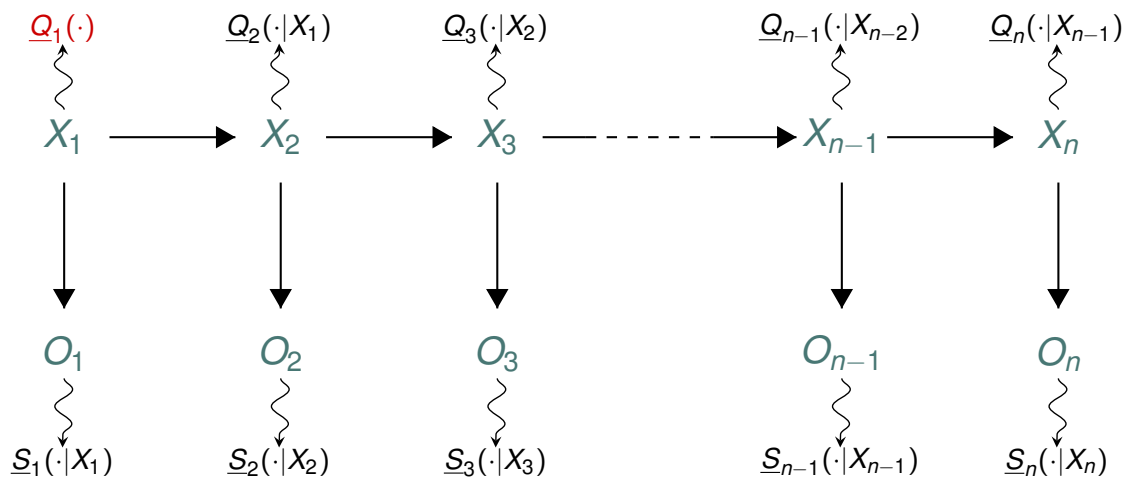

imprecise emission models 


\section{Imprecise hidden Markov model}

We consider stationary imprecise hidden Markov models

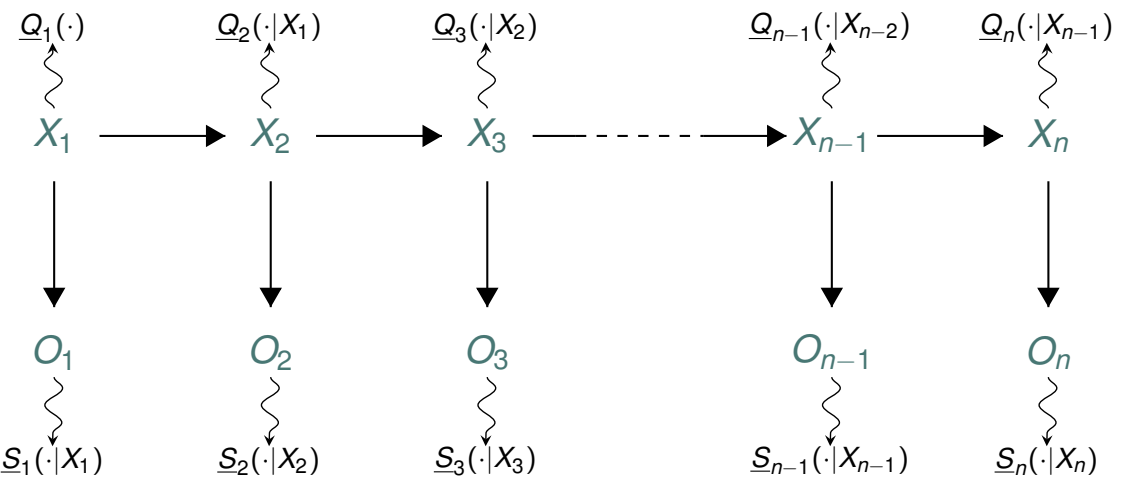




\section{Imprecise hidden Markov model}

We consider stationary imprecise hidden Markov models

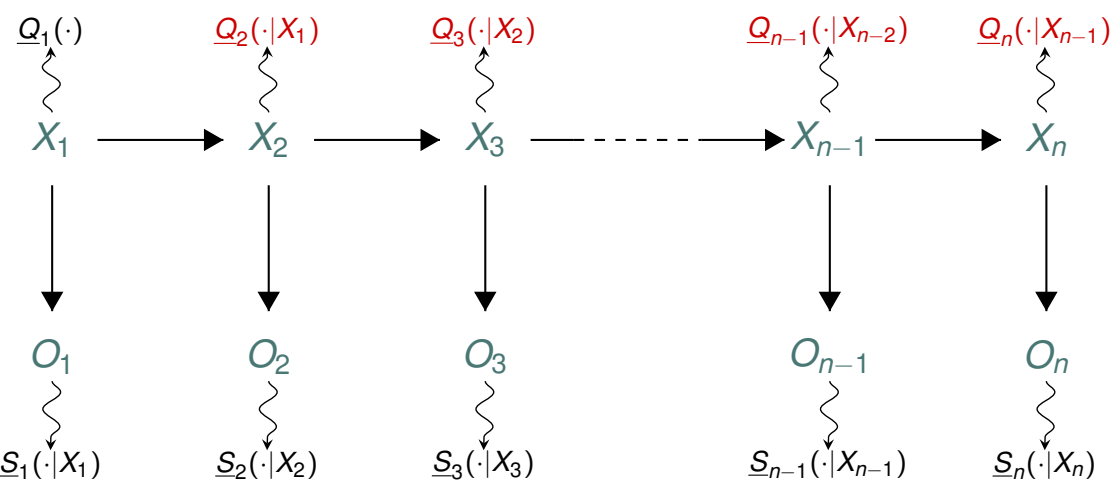




\section{Imprecise hidden Markov model}

We consider stationary imprecise hidden Markov models
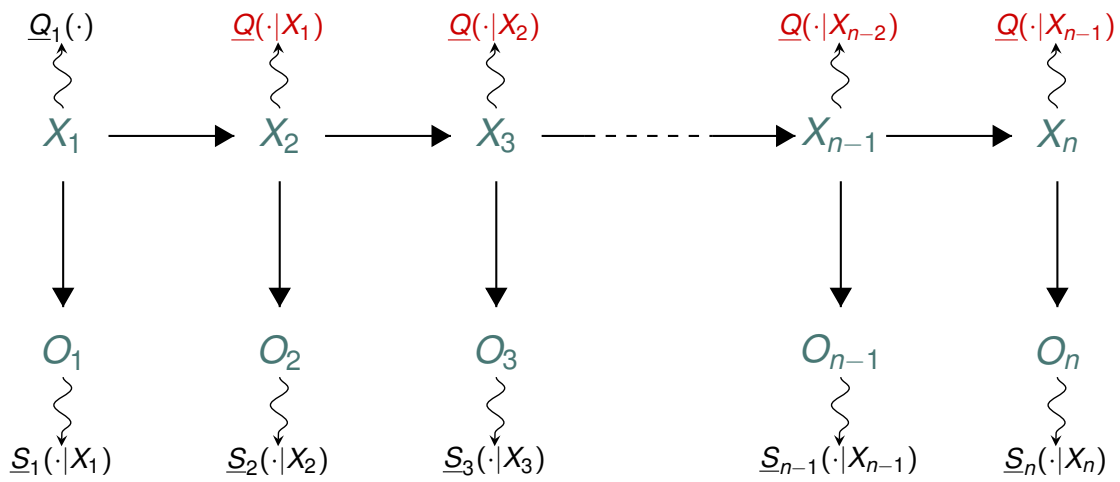

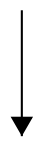

$O_{n-1}$

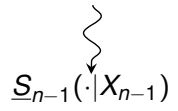

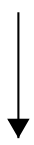

$O_{n}$

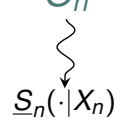




\section{Imprecise hidden Markov model}

We consider stationary imprecise hidden Markov models
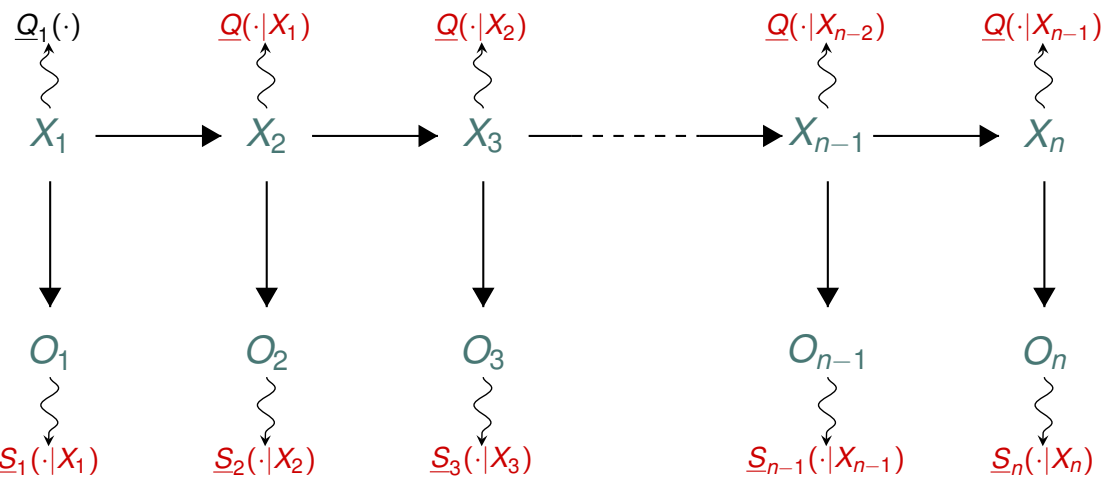


\section{Imprecise hidden Markov model}

We consider stationary imprecise hidden Markov models

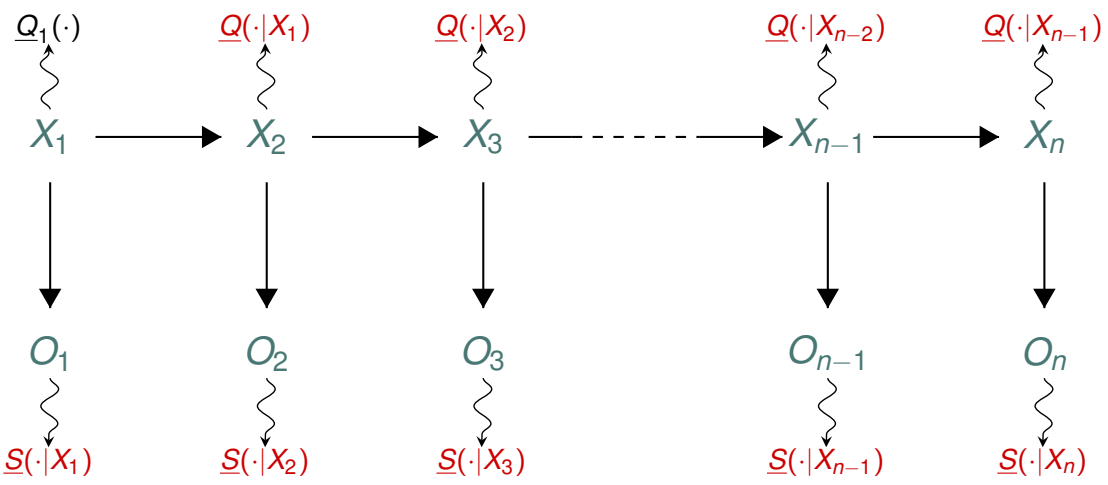


What do we want to do? 


\section{Our problem of interest}

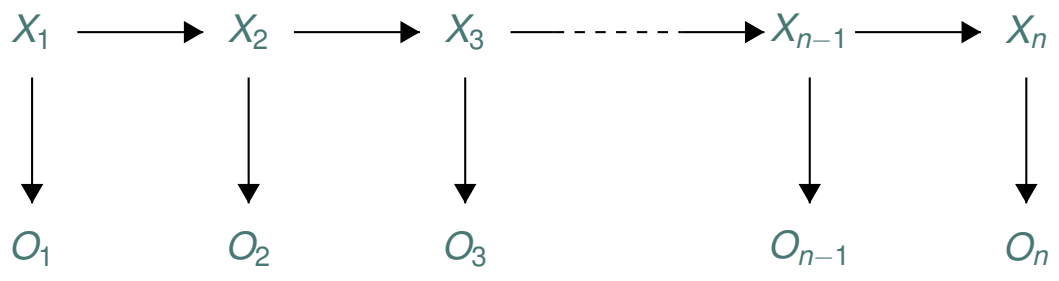




\section{Our problem of interest}

Suppose we know the output sequence

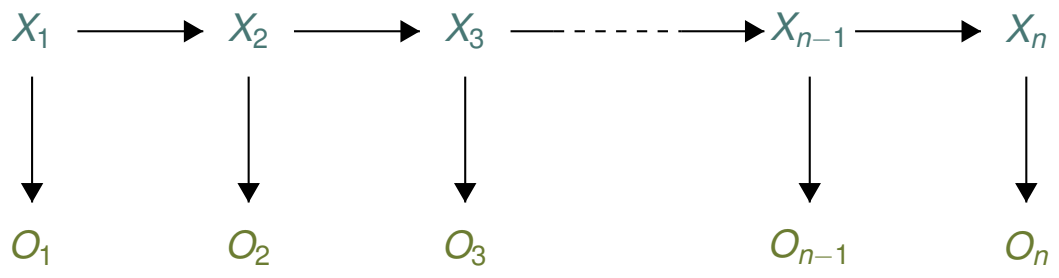




\section{Our problem of interest}

Suppose we know the output sequence: $O_{1: n}=0_{1: n} \in \mathscr{O}^{n}$

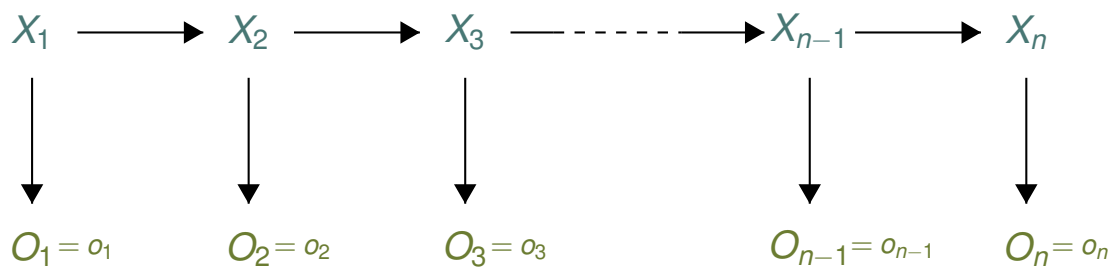




\section{Our problem of interest}

Suppose we know the output sequence: $O_{1: n}=0_{1: n} \in \mathscr{O}^{n}$, we want to estimate the unknown local uncertainty models.

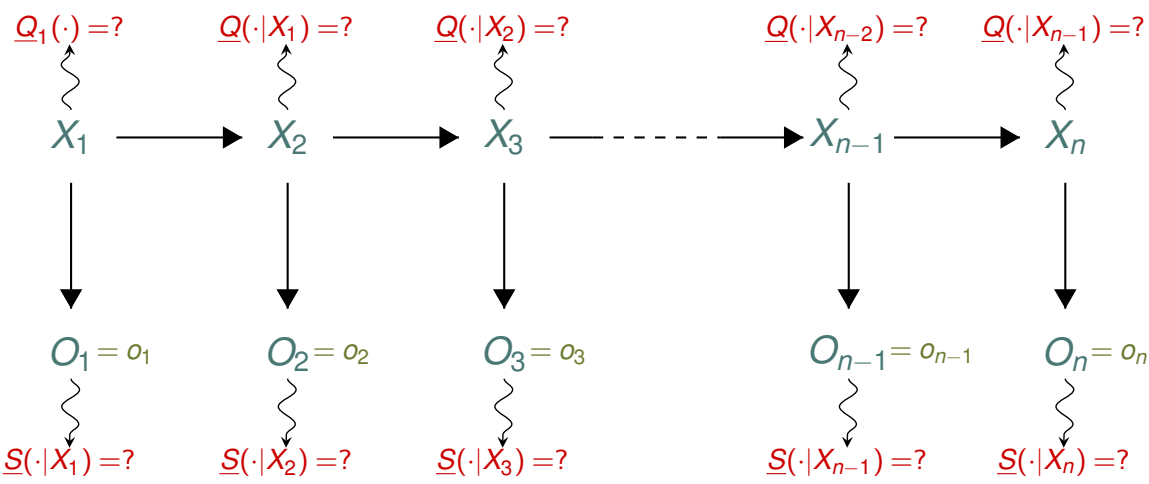


How could you learn the local models if the state sequence were known? 


\section{An easier problem}

We know the output sequence: $O_{1: n}=0_{1: n} \in \mathscr{O}^{n}$.

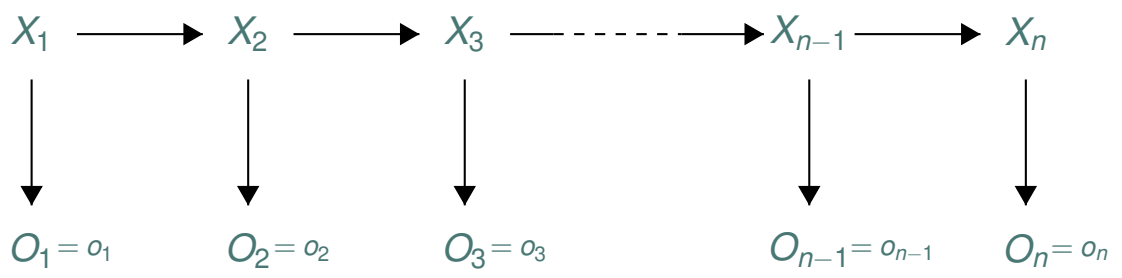




\section{An easier problem}

We know the output sequence: $O_{1: n}=O_{1: n} \in \mathscr{O}^{n}$.

Suppose we know in addition also the state sequence: $X_{1: n}=x_{1: n} \in \mathscr{X}^{n}$

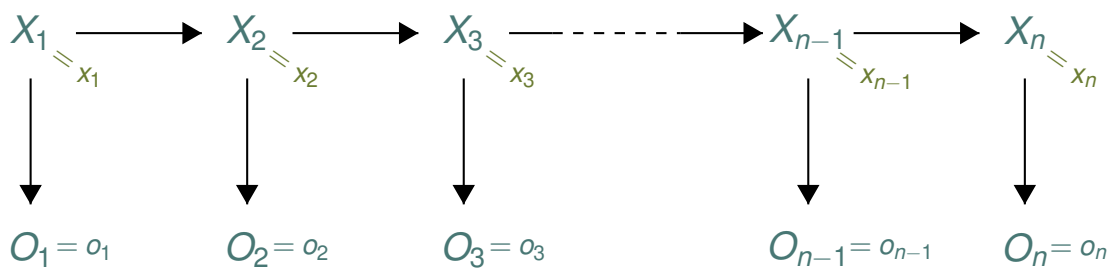




\section{An easier problem}

We know the output sequence: $O_{1: n}=o_{1: n} \in \mathscr{O}^{n}$.

Suppose we know in addition also the state sequence: $x_{1: n}=x_{1: n} \in \mathscr{X}^{n}$, how can we learn local models now?

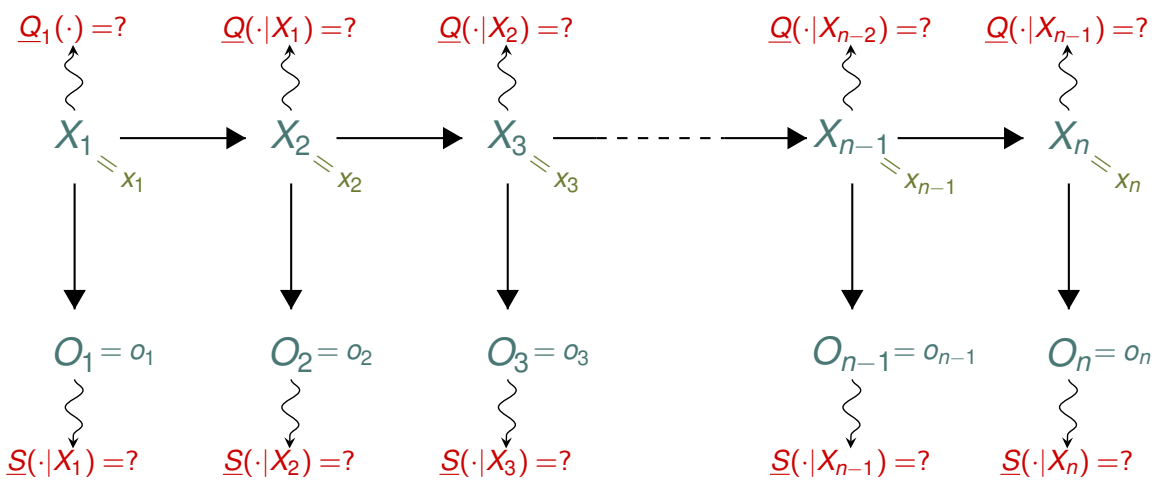




\section{Solution}

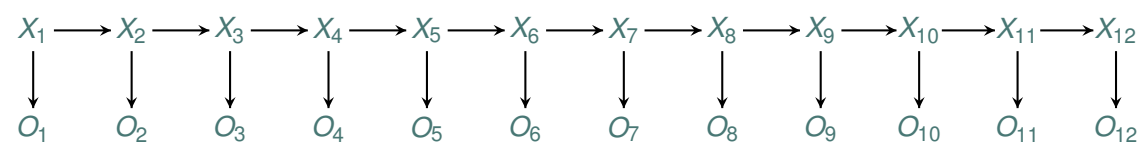




\section{Solution}

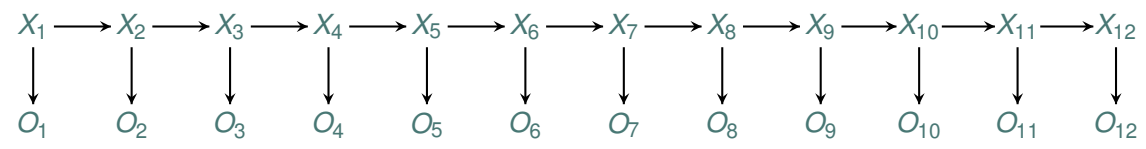

Suppose $\mathscr{X}=\{a, b\}$ and $\mathscr{O}=\{\boldsymbol{o}, \boldsymbol{p}, \boldsymbol{q}\}$. 


\section{Solution}

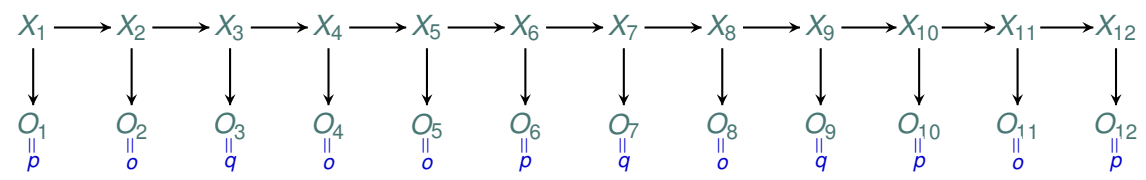

Suppose $\mathscr{X}=\{a, b\}$ and $\mathscr{O}=\{o, p, q\}$. 


\section{Solution}

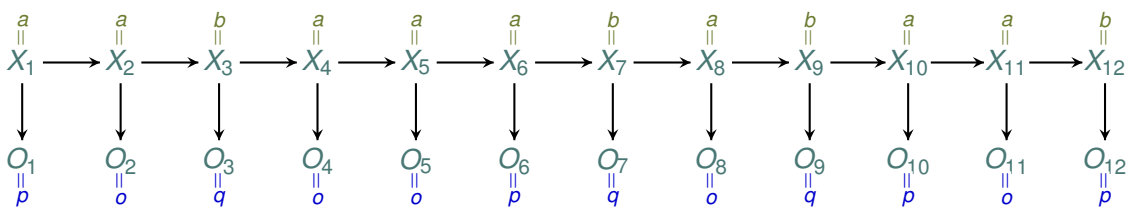

Suppose $\mathscr{X}=\{a, b\}$ and $\mathscr{O}=\{o, p, q\}$. 


\section{Solution}

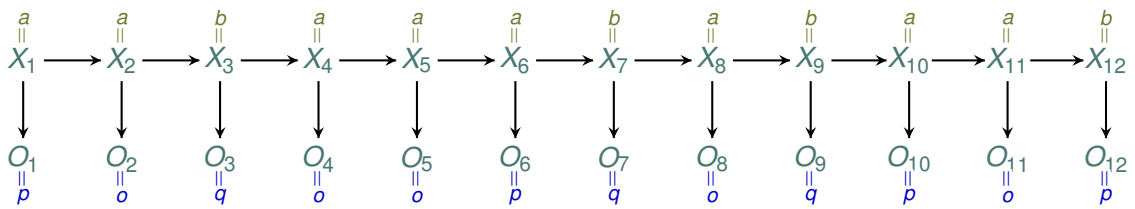

Suppose $\mathscr{X}=\{a, b\}$ and $\mathscr{O}=\{o, p, q\}$.

With (known) hidden state sequence $x_{1: n}$ and output sequence $0_{1: n}$ $(x, y \in \mathscr{X}$ and $z \in \mathscr{O})$ :

$n_{x}$ : number of times a state $x$ is reached,

$n_{x, y}$ : number of times a state transition from $x$ to $y$ takes place,

$n_{x, z}$ : number of times a state $x$ emits an output $z$. 


\section{Solution}

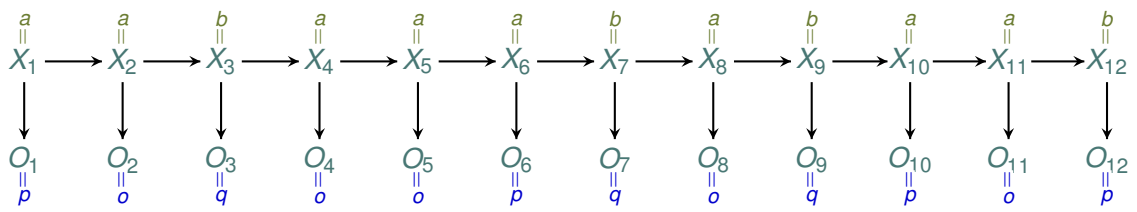

Suppose $\mathscr{X}=\{a, b\}$ and $\mathscr{O}=\{o, p, q\}$.

With (known) hidden state sequence $x_{1: n}$ and output sequence $0_{1: n}$ $(x, y \in \mathscr{X}$ and $z \in \mathscr{O})$ :

$n_{x}$ : number of times a state $x$ is reached,

$n_{x, y}$ : number of times a state transition from $x$ to $y$ takes place,

$n_{x, z}$ : number of times a state $x$ emits an output $z$.

Here:

$$
\left.\begin{array}{r}
n_{a}=8, n_{b}=4, \\
n_{a, a}=4, n_{a, b}=4, n_{b, a}=3, n_{b, b}=0, \\
n_{a, o}=5, n_{a, p}=3, n_{a, q}=0, \\
n_{b, o}=0, n_{b, p}=1, n_{b, q}=3 .
\end{array}\right\}
$$




\section{Solution}

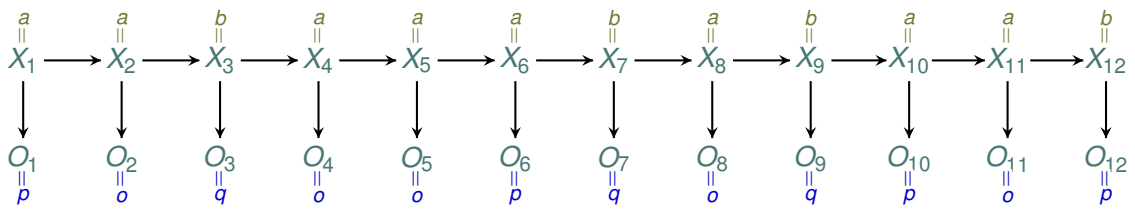

Suppose $\mathscr{X}=\{a, b\}$ and $\mathscr{O}=\{o, p, q\}$.

With (known) hidden state sequence $x_{1: n}$ and output sequence $0_{1: n}$ $(x, y \in \mathscr{X}$ and $z \in \mathscr{O})$ :

$n_{x}$ : number of times a state $x$ is reached,

$n_{x, y}$ : number of times a state transition from $x$ to $y$ takes place,

$n_{x, z}$ : number of times a state $x$ emits an output $z$.

Here:

$$
\left.\begin{array}{r}
n_{a}=8, n_{b}=4, \\
n_{a, a}=4, n_{a, b}=4, n_{b, a}=3, n_{b, b}=0, \\
n_{a, o}=5, n_{a, p}=3, n_{a, q}=0, \\
n_{b, o}=0, n_{b, p}=1, n_{b, q}=3 .
\end{array}\right\} \begin{aligned}
& \text { With these counts, how } \\
& \text { can we build local } \\
& \text { models? }
\end{aligned}
$$




\section{Imprecise Dirichlet model}

We use the imprecise Dirichlet model (IDM) to compute estimates for the local models. If $n(A)$ is the number of occurences of an event $A$ in $N$ experiments, then the lower and upper probability of $A$ according to an IDM are defined as

$$
\underline{P}(A)=\frac{n(A)}{s+N} \quad \text { and } \quad \bar{P}(A)=\frac{s+n(A)}{s+N} .
$$

$s>0$ is the number of pseudo-counts, which is an inverse measure of the speed of convergence to a precise model. 


\section{Imprecise Dirichlet model}

We use the imprecise Dirichlet model (IDM) to compute estimates for the local models. If $n(A)$ is the number of occurences of an event $A$ in $N$ experiments, then the lower and upper probability of $A$ according to an IDM are defined as

$$
\underline{P}(A)=\frac{n(A)}{s+N} \quad \text { and } \quad \bar{P}(A)=\frac{s+n(A)}{s+N} .
$$

$s>0$ is the number of pseudo-counts, which is an inverse measure of the speed of convergence to a precise model.

Now, we use the quantities $n_{x}, n_{x, y}$ and $n_{x, z}$ (with $x, y \in \mathscr{X}$ and $z \in \mathscr{O}$ ) to estimate the imprecise transition and emission models: 


\section{Imprecise Dirichlet model}

We use the imprecise Dirichlet model (IDM) to compute estimates for the local models. If $n(A)$ is the number of occurences of an event $A$ in $N$ experiments, then the lower and upper probability of $A$ according to an IDM are defined as

$$
\underline{P}(A)=\frac{n(A)}{s+N} \quad \text { and } \quad \bar{P}(A)=\frac{s+n(A)}{s+N} .
$$

$s>0$ is the number of pseudo-counts, which is an inverse measure of the speed of convergence to a precise model.

Now, we use the quantities $n_{x}, n_{x, y}$ and $n_{x, z}$ (with $x, y \in \mathscr{X}$ and $z \in \mathscr{O}$ ) to estimate the imprecise transition and emission models:

$$
\begin{gathered}
\underline{Q}(\{y\} \mid x)=\frac{n_{x, y}}{s+\sum_{y^{*} \in \mathscr{X}} n_{x, y^{*}}} \quad \text { and } \quad \bar{Q}(\{y\} \mid x)=\frac{s+n_{x, y}}{s+\sum_{y^{*} \in \mathscr{X}} n_{x, y^{*}}}, \\
\underline{S}(\{z\} \mid x)=\frac{n_{x, z}}{s+n_{x}} \quad \text { and } \quad \bar{S}(\{z\} \mid x)=\frac{s+n_{x, z}}{s+n_{x}}
\end{gathered}
$$




\section{Example}

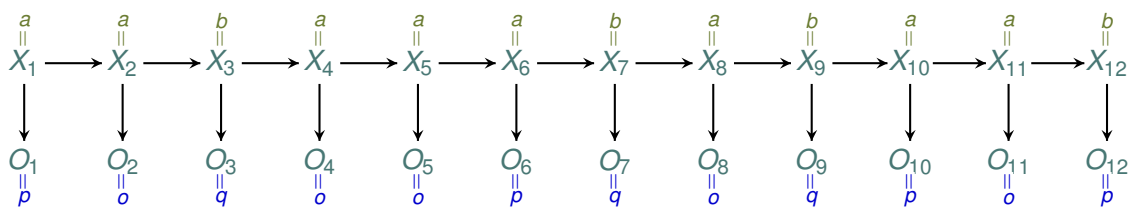

(with $x, y \in \mathscr{X}$ and $z \in \mathscr{O}$ ):

$\underline{Q}(\{y\} \mid x)=\frac{n_{x, y}}{s+\sum_{y^{*} \in \mathscr{X}} n_{x, y^{*}}}, \bar{Q}(\{y\} \mid x)=\frac{s+n_{x, y}}{s+\sum_{y^{*} \in \mathscr{X}} n_{x, y^{*}}}, \underline{S}(\{z\} \mid x)=\frac{n_{x, z}}{s+n_{x}}, \bar{S}(\{z\} \mid x)=\frac{s+n_{x, z}}{s+n_{x}}$. 


\section{Example}

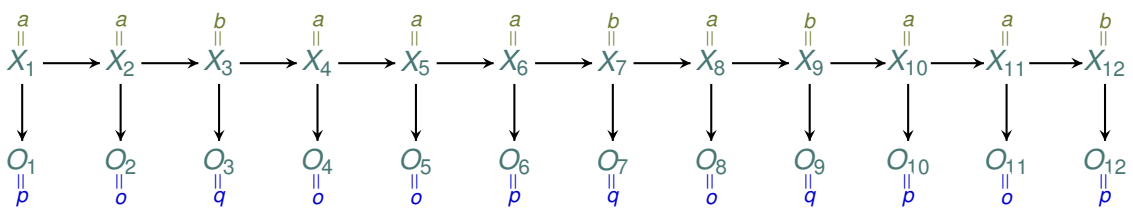

(with $x, y \in \mathscr{X}$ and $z \in \mathscr{O}$ ):

$\underline{Q}(\{y\} \mid x)=\frac{n_{x, y}}{s+\sum_{y^{*} \in \mathscr{X}} n_{x, y^{*}}}, \bar{Q}(\{y\} \mid x)=\frac{s+n_{x, y}}{s+\sum_{y^{*} \in \mathscr{X}} n_{x, y^{*}}}, \underline{S}(\{z\} \mid x)=\frac{n_{x, z}}{s+n_{x}}, \bar{S}(\{z\} \mid x)=\frac{s+n_{x, z}}{s+n_{x}}$.

Here, with $s=2$ :

$\underline{Q}(\{a\} \mid a)=2 / 5$

$\bar{Q}(\{a\} \mid a)=3 / 5, \quad \underline{Q}(\{b\} \mid a)=2 / 5$,

$\bar{Q}(\{b\} \mid a)=3 / 5$

$\underline{Q}(\{a\} \mid b)=3 / 5$

$\bar{Q}(\{a\} \mid b)=1$,

$\underline{Q}(\{b\} \mid b)=0$,

$\bar{Q}(\{b\} \mid b)=2 / 5$,

$\underline{S}(\{\boldsymbol{o}\} \mid a)=1 / 2$

$\bar{S}(\{0\} \mid a)=7 / 10$

$\underline{S}(\{0\} \mid b)=0$,

$\bar{S}(\{0\} \mid b)=1 / 3$,

$\underline{S}(\{\boldsymbol{p}\} \mid a)=3 / 10$

$\bar{S}(\{\boldsymbol{p}\} \mid a)=1 / 2$

$S(\{p\} \mid b)=1 / 6$,

$\bar{S}(\{\boldsymbol{p}\} \mid b)=1 / 2$,

$\underline{S}(\{q\} \mid a)=0$,

$\bar{S}(\{\boldsymbol{q}\} \mid a)=1 / 5, \quad \underline{S}(\{\boldsymbol{q}\} \mid b)=1 / 5$,

$\bar{S}(\{q\} \mid b)=3 / 5$. 


\section{But the state sequence is hidden...}




\section{We are almost there}

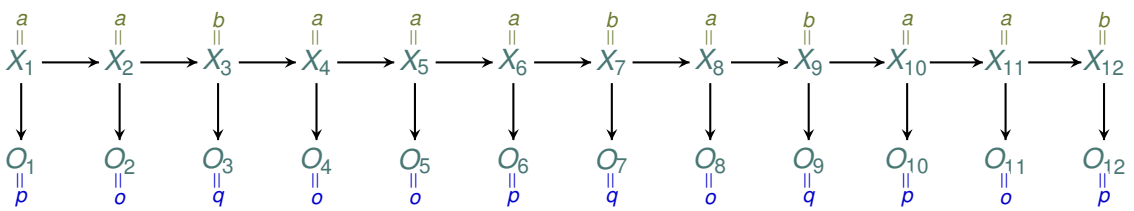




\section{We are almost there}

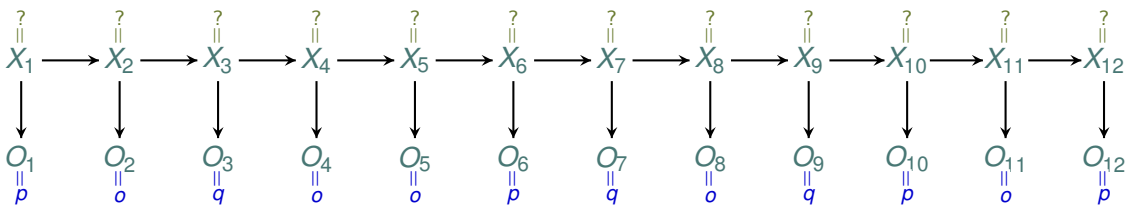




\section{We are almost there}

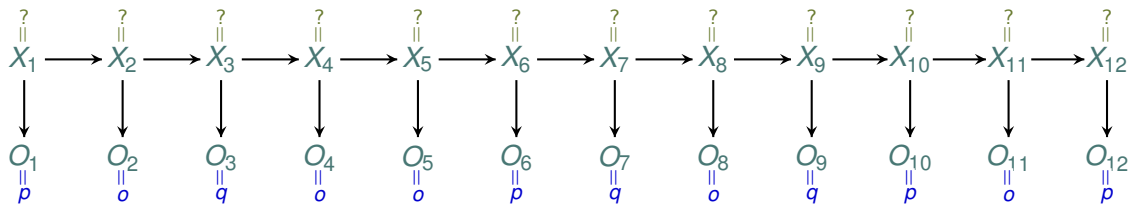

The state sequence $x_{1: n} \in \mathscr{X}^{n}$ is hidden, so it is a random variable $X_{1: n}$. 


\section{We are almost there}

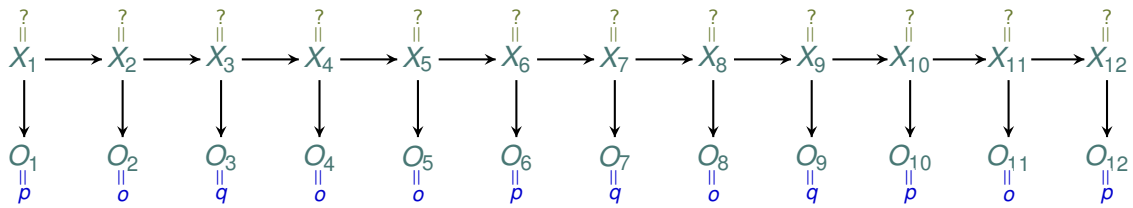

The state sequence $x_{1: n} \in \mathscr{X}^{n}$ is hidden, so it is a random variable $X_{1: n}$. (with $x, y \in \mathscr{X}$ and $z \in \mathscr{O}$ ) $n_{x}, n_{x, y}$ and $n_{x, z}$ are random variables $N_{x}, N_{x, y}$ and $N_{x, z}$. 


\section{We are almost there}

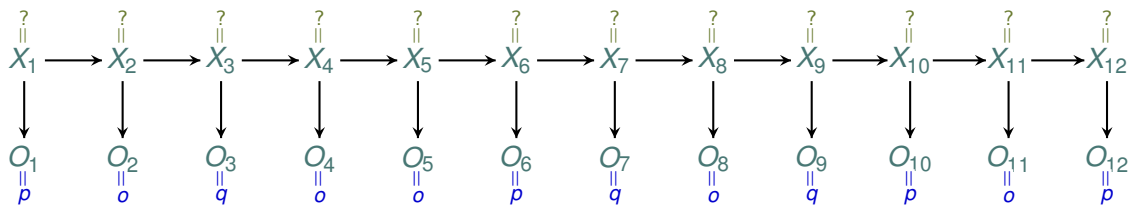

The state sequence $x_{1: n} \in \mathscr{X}^{n}$ is hidden, so it is a random variable $X_{1: n}$. (with $x, y \in \mathscr{X}$ and $z \in \mathscr{O}$ ) $n_{x}, n_{x, y}$ and $n_{x, z}$ are random variables $N_{x}, N_{x, y}$ and $N_{x, z}$.

Idea: instead of using real counts, use estimates:

$$
\begin{gathered}
\hat{n}_{x}, \\
\hat{n}_{x, y}, \\
\hat{n}_{x, z} .
\end{gathered}
$$




\section{We are almost there}

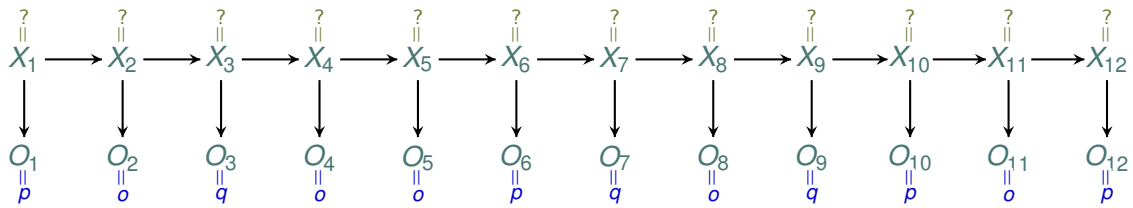

The state sequence $x_{1: n} \in \mathscr{X}^{n}$ is hidden, so it is a random variable $X_{1: n}$. (with $x, y \in \mathscr{X}$ and $z \in \mathscr{O}$ ) $n_{x}, n_{x, y}$ and $n_{x, z}$ are random variables $N_{x}, N_{x, y}$ and $N_{x, z}$.

Idea: instead of using real counts, use expected counts:

$$
\begin{aligned}
\hat{n}_{x} & =E\left(N_{x} \mid o_{1: n}, \theta^{*}\right), \\
\hat{n}_{x, y} & =E\left(N_{x, y} \mid 0_{1: n}, \theta^{*}\right), \\
\hat{n}_{x, z} & =E\left(N_{x, z} \mid 0_{1: n}, \theta^{*}\right) .
\end{aligned}
$$




\section{We are almost there}

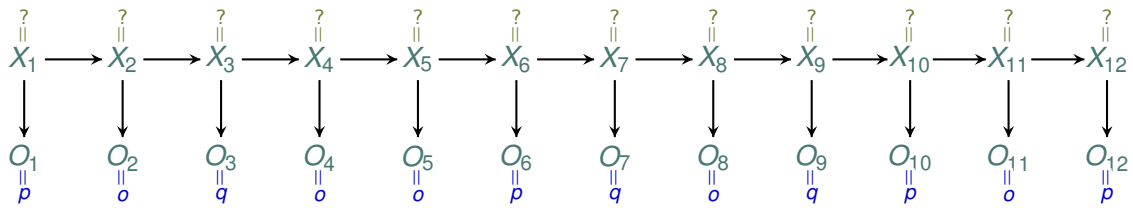

The state sequence $x_{1: n} \in \mathscr{X}^{n}$ is hidden, so it is a random variable $X_{1: n}$. (with $x, y \in \mathscr{X}$ and $z \in \mathscr{O}$ ) $n_{x}, n_{x, y}$ and $n_{x, z}$ are random variables $N_{x}, N_{x, y}$ and $N_{x, z}$.

Idea: instead of using real counts, use expected counts

$$
\begin{aligned}
\hat{n}_{x} & =E\left(N_{x} \mid o_{1: n}, \theta^{*}\right), \\
\hat{n}_{x, y} & =E\left(N_{x, y} \mid 0_{1: n}, \theta^{*}\right), \\
\hat{n}_{x, z} & =E\left(N_{x, z} \mid 0_{1: n}, \theta^{*}\right) .
\end{aligned}
$$

$O_{1: n}$ is the known output sequence, and $\theta^{*}$ represents the model parameter. 


\section{We are almost there}

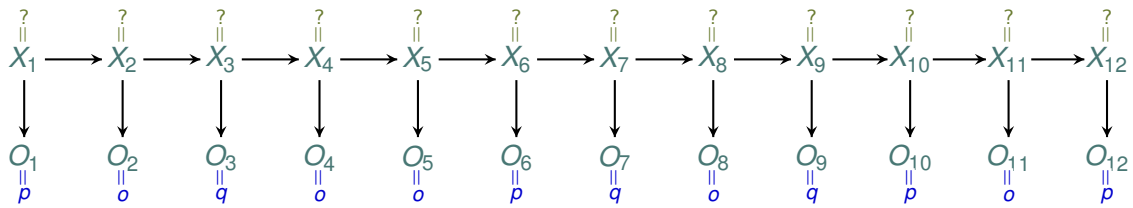

The state sequence $x_{1: n} \in \mathscr{X}^{n}$ is hidden, so it is a random variable $X_{1: n}$. (with $x, y \in \mathscr{X}$ and $z \in \mathscr{O}$ ) $n_{x}, n_{x, y}$ and $n_{x, z}$ are random variables $N_{x}, N_{x, y}$ and $N_{x, z}$.

Idea: instead of using real counts, use expected counts

$$
\begin{aligned}
\hat{n}_{x} & =E\left(N_{x} \mid o_{1: n}, \theta^{*}\right), \\
\hat{n}_{x, y} & =E\left(N_{x, y} \mid 0_{1: n}, \theta^{*}\right), \\
\hat{n}_{x, z} & =E\left(N_{x, z} \mid 0_{1: n}, \theta^{*}\right) .
\end{aligned}
$$

$O_{1: n}$ is the known output sequence, and $\theta^{*}$ represents the model parameter. We can calculate $\theta^{*}$ with the Baum-Welch algorithm, so the idea makes sense. 


\section{Estimated local models}

With known state sequence $x_{1: n}(x, y \in \mathscr{X}$ and $z \in \mathscr{O})$ :

$$
\begin{gathered}
\underline{Q}(\{y\} \mid x)=\frac{n_{x, y}}{s+\sum_{y^{*} \in \mathscr{X}} n_{x, y^{*}}} \text { and } \bar{Q}(\{y\} \mid x)=\frac{s+n_{x, y}}{s+\sum_{y^{*} \in \mathscr{X}} n_{x, y^{*}}}, \\
\underline{S}(\{z\} \mid x)=\frac{n_{x, z}}{s+n_{x}} \text { and } \bar{S}(\{z\} \mid x)=\frac{s+n_{x, z}}{s+n_{x}} .
\end{gathered}
$$




\section{Estimated local models}

With unknown state sequence $X_{1: n}(x, y \in \mathscr{X}$ and $z \in \mathscr{O})$ :

$$
\begin{gathered}
\underline{Q}(\{y\} \mid x)=\frac{\hat{n}_{x, y}}{s+\sum_{y^{*} \in \mathscr{X}} \hat{n}_{x, y^{*}}} \text { and } \bar{Q}(\{y\} \mid x)=\frac{s+\hat{n}_{x, y}}{s+\sum_{y^{*} \in \mathscr{X}} \hat{n}_{x, y^{*}}}, \\
\underline{S}(\{z\} \mid x)=\frac{\hat{n}_{x, z}}{s+\hat{n}_{x}} \text { and } \bar{S}(\{z\} \mid x)=\frac{s+\hat{n}_{x, z}}{s+\hat{n}_{x}} .
\end{gathered}
$$




\section{Example: predicting future earthquake rates}

We want to predict future earthquake rates, based on number of earthquakes in previous years. 


\section{Example: predicting future earthquake rates}

We want to predict future earthquake rates, based on number of earthquakes in previous years.

Assumptions:

- Earth can be in 3 possible seismic states $\lambda_{1}, \lambda_{2}$ and $\lambda_{3}$, 


\section{Example: predicting future earthquake rates}

We want to predict future earthquake rates, based on number of earthquakes in previous years.

\section{Assumptions:}

- Earth can be in 3 possible seismic states $\lambda_{1}, \lambda_{2}$ and $\lambda_{3}$,

- occurrence of earthquakes in a year depends on the seismic state in that year, 


\section{Example: predicting future earthquake rates}

We want to predict future earthquake rates, based on number of earthquakes in previous years.

\section{Assumptions:}

- Earth can be in 3 possible seismic states $\lambda_{1}, \lambda_{2}$ and $\lambda_{3}$,

- occurrence of earthquakes in a year depends on the seismic state in that year,

- Earth in state $\lambda$ emits $O$ earthquakes in a year. 


\section{Example: predicting future earthquake rates}

We want to predict future earthquake rates, based on number of earthquakes in previous years.

\section{Assumptions:}

- Earth can be in 3 possible seismic states $\lambda_{1}, \lambda_{2}$ and $\lambda_{3}$,

- occurrence of earthquakes in a year depends on the seismic state in that year,

- Earth in state $\lambda$ emits $O$ earthquakes in a year.

We model our problem as an imprecise hidden Markov model. 


\section{Example: predicting future earthquake rates}

We want to predict future earthquake rates, based on number of earthquakes in previous years.

\section{Assumptions:}

- Earth can be in 3 possible seismic states: $\mathscr{X}=\left\{\lambda_{1}, \lambda_{2}, \lambda_{3}\right\}$,

- occurrence of earthquakes in a year depends on the seismic state in that year,

- Earth in state $\lambda$ emits $O$ earthquakes in a year.

We model our problem as an imprecise hidden Markov model. 


\section{Example: predicting future earthquake rates}

We want to predict future earthquake rates, based on number of earthquakes in previous years.

\section{Assumptions:}

- Earth can be in 3 possible seismic states: $\mathscr{X}=\left\{\lambda_{1}, \lambda_{2}, \lambda_{3}\right\}$,

- occurrence of earthquakes in a year depends on the seismic state in that year,

- Earth in state $\lambda$ emits $O$ earthquakes in a year: $\mathscr{O}=\mathbb{N} \cup\{0\}$ and emission model $S(o \mid \lambda)$ is a Poisson process, represented by the precise probability mass function $p(o \mid \lambda)=\frac{e^{-\lambda} \lambda^{0}}{0 !}$.

We model our problem as an imprecise hidden Markov model. 


\section{Example: predicting future earthquake rates}

We want to predict future earthquake rates, based on number of earthquakes in previous years.

\section{Assumptions:}

- Earth can be in 3 possible seismic states: $\mathscr{X}=\left\{\lambda_{1}, \lambda_{2}, \lambda_{3}\right\}$,

- occurrence of earthquakes in a year depends on the seismic state in that year,

- Earth in state $\lambda$ emits $O$ earthquakes in a year: $\mathscr{O}=\mathbb{N} \cup\{0\}$ and emission model $S(o \mid \lambda)$ is a Poisson process, represented by the precise probability mass function $p(o \mid \lambda)=\frac{e^{-\lambda} \lambda^{0}}{0 !}$.

We model our problem as an imprecise hidden Markov model.

Our observation: number of earthquakes from 1900 to 2006 


\section{Example: learned model}

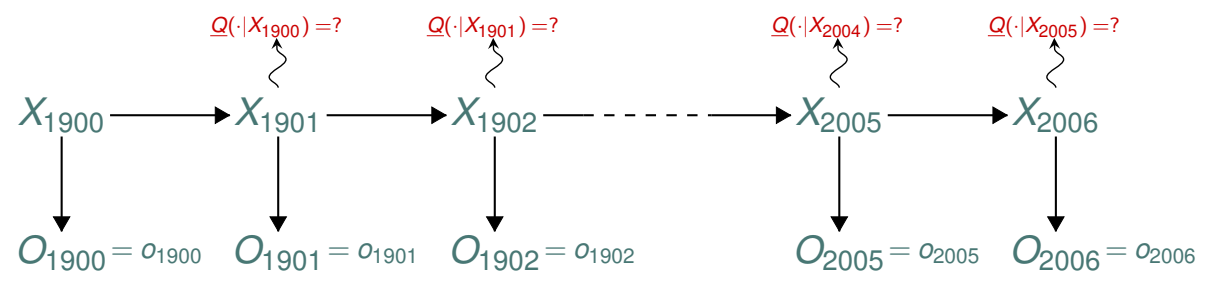

Based on the data, we learn the (imprecise) transition model. 


\section{Example: learned model}

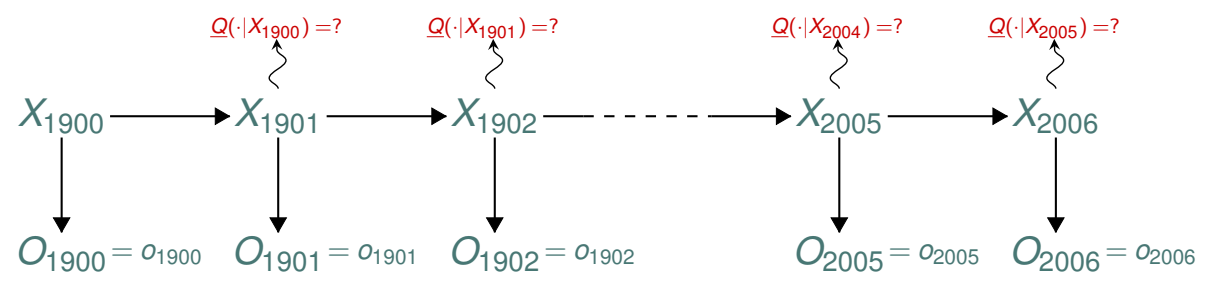

Based on the data, we learn the (imprecise) transition model.

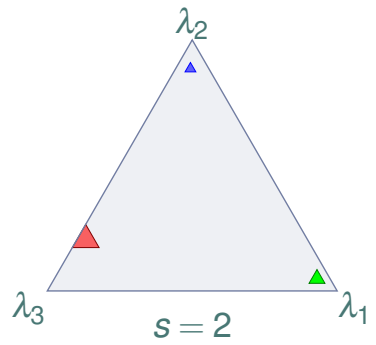

$\square \underline{Q}\left(\cdot \mid \lambda_{1}\right)$

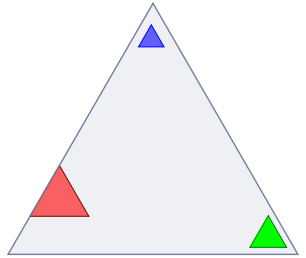

$s=5$

$\square \underline{Q}\left(\cdot \mid \lambda_{2}\right)$

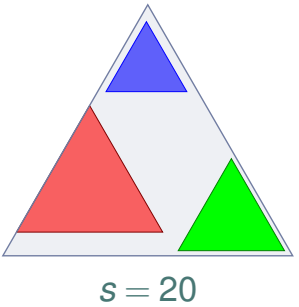

$Q\left(\cdot \mid \lambda_{3}\right)$ 


\section{Example: predicting earthquake rates}

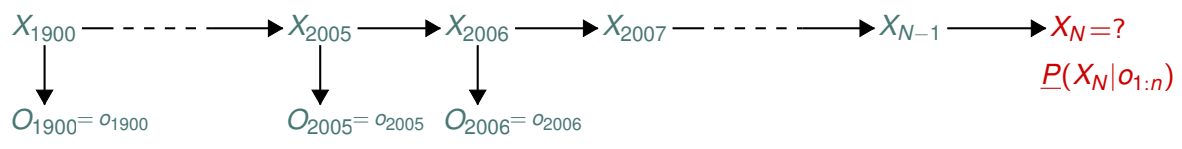

With the learned imprecise hidden Markov model, we predict future earthquake rates. We use the MePiCTIr algorithm (de Cooman et al., 2010). 


\section{Example: predicting earthquake rates}

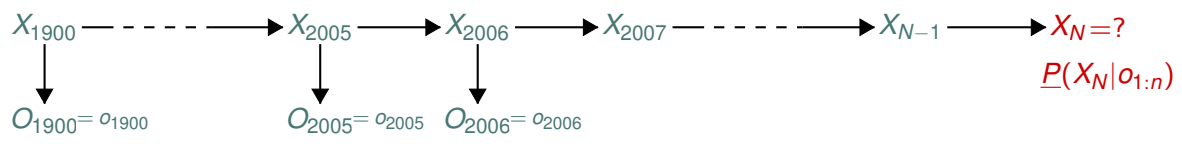

With the learned imprecise hidden Markov model, we predict future earthquake rates. We use the MePiCTIr algorithm (de Cooman et al., 2010).
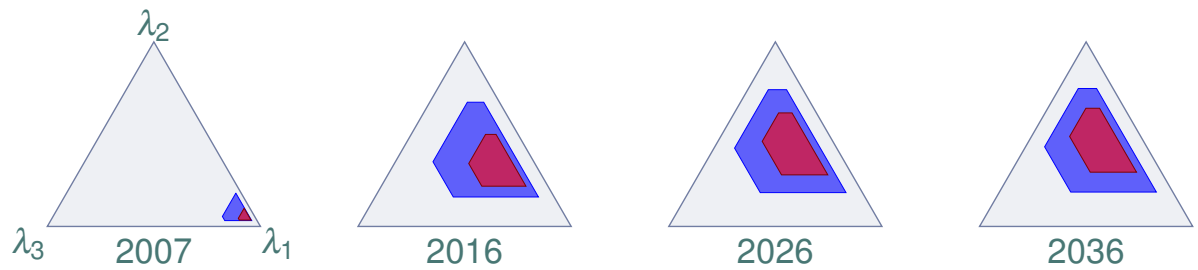

$\square \quad s=2$

$\square=5$ 